\section{D Printing on Textiles: Testing of Adhesion}

Malengier $B^{1^{*}}$, Hertleer $C^{1}$, Cardon $L^{2}$ and Van Langenhove $L^{1}$

\begin{abstract}
3D printing on textiles is a possible future application for mass customization of fabrics and textile products. In this paper we investigate three test methods to quantify the adhesion of $3 \mathrm{D}$ printed PLA parts to a textile substrate. The first proposed test method is a perpendicular tensile test, the second is a shear test, and the third is a peel test. These three tests are used to characterise the adhesion of a 3D printed shape on six different textile substrates. The test methods are compared and it is shown how they can help in standardizing the research of 3D printing on textiles.
\end{abstract}

\section{Keywords}

Adhesion; 3D printing; Textile; Tensile test; Shear test; Peel test

\section{Introduction}

3D printing, also known as Additive Manufacturing (AM), describes a set of techniques where an object is constructed layer-bylayer [1]. This technique has become quite popular because of its many advantages, such as flexibility and ease of use. Also the combination with textile materials as substrate is possible and was quickly recognized [2-4]. 3D printing on textiles is a possible innovative production method that allows new applications, as well as dedicated customization of a common base fabric, with a minimal investment cost and a wide range of possibilities. In this context the question arises how good the $3 \mathrm{D}$ printed object adheres to the textile substrate, as this will contribute to the perception of better or worse quality of the overall product.

In this paper three test methods to quantify the adhesion of a 3D printed object to a textile substrate are proposed and investigated. As printing method the extrusion based added manufacturing technique - also known as Fused Deposition Modeling (FDM) - is used via a Felix low cost 3D-printer.

An FDM printer extrudes the material with use of a nozzle and heat. The printing material is melted and deposited selectively and line wise on the printer bed, so that one layer is formed. Subsequently, the printer bed is lowered and a new layer is deposited. In our test setup, a standard PLA filament is used for printing and a textile substrate is attached to the printer bed so that the $3 \mathrm{D}$ printed object is formed on the textile substrate.

Not every textile material can provide a good adhesion of the $3 \mathrm{D}$ printed part. Hence, dedicated research is required to determine the

*Corresponding author: Malengier Benny, Centre for Textile Science and Engineering, Department MaTCh, Ghent University, Gent, Belgium, Tel: + 09 26454 07; E-mail: benny.malengier@ugent.be

Received: December 04, 2017 Accepted: February 28, 2018 Published: March 05, 2018 adhesion properties and process parameters that give the best results [5]. However, so far, no normalized test method is available to make this evaluation. In Section 2 three test methods are proposed: a tensile test, a shear test and a peel test, all are suitable for testing the adhesion of $3 \mathrm{D}$ prints on textiles. These can be used with different combinations of substrates and 3D printing techniques. In Section 3 we list the materials used in evaluating these adhesion tests. Finally, Section 4 gives the results of this study.

\section{Adhesion Tests}

To select test methods that enable the evaluation of the adhesion properties between a 3D printed object and a textile substrate, we were inspired by existing test methods that have the purpose of characterising the adhesion between two materials. At first a study was done of available standards. After extensive testing, we suggest the following three test methods for 3D printed objects. Prior to performing these tests, a well-defined shape is $3 \mathrm{D}$ printed on the textile substrate.

\section{Perpendicular tensile test}

The first proposed test method for an accurate characterisation of the test sample is a perpendicular tensile test. This test uses a pawn shaped object, called "a dolly", which is directly printed onto the textile substrate. This textile substrate, together with the dolly are both clamped onto a tensile frame, as shown in Figure 1 schematically, and visible in the clamp in Figure 2 to the right. The maximum force needed to detach the dolly from the textiles is measured by the tensile tester. Obviously the higher the required force, the better the adhesion.

\section{Shear test}

A second proposed test was based on the standard EN $1373^{1}$ which is a European Standard that specifies a test method to measure the adhesion of a resilient or textile floorcovering or wall covering bonded to a given substrate under shear forces. (The term "wall covering" does not include any type of wallpaper). To perform the shear test in a framework of this research, a rectangular plate is printed onto the textile substrate. Its dimensions are a length of $60 \mathrm{~mm}$, a width of 50 $\mathrm{mm}$ and a height of $4 \mathrm{~mm}$. There is an overlap between the textile and the printed plate of $30 \mathrm{~mm}$, as can be seen in Figure 1 Right.

The specimen was placed in the tensile frame with the lower clamp holding the textile and the upper clamp taking the printed plate. The gauge length between the clamps is set to $100 \mathrm{~mm}$, as shown in Figure 1-Right. While testing, the clamps moved away with a crosshead movement of $50 \mathrm{~mm} / \mathrm{min}$. The tensile frame measures the maximum force that can be applied before destroying the specimen.

\section{Peel test}

Finally, a third proposed test evolved from the previous shear test: a peel test. The setup of the peel test and the dimensions of the specimen were identical to the setup of the shear test. The difference however, lies in the placement of the 3D printed object on the textile substrate. The tensile frame measures the maximum force needed to detach the fabric from the 3D printed object (Figure 3).

1 EN 1373:1999 Adhesives. Test method for adhesives for floor and wall coverings. Shear test. 


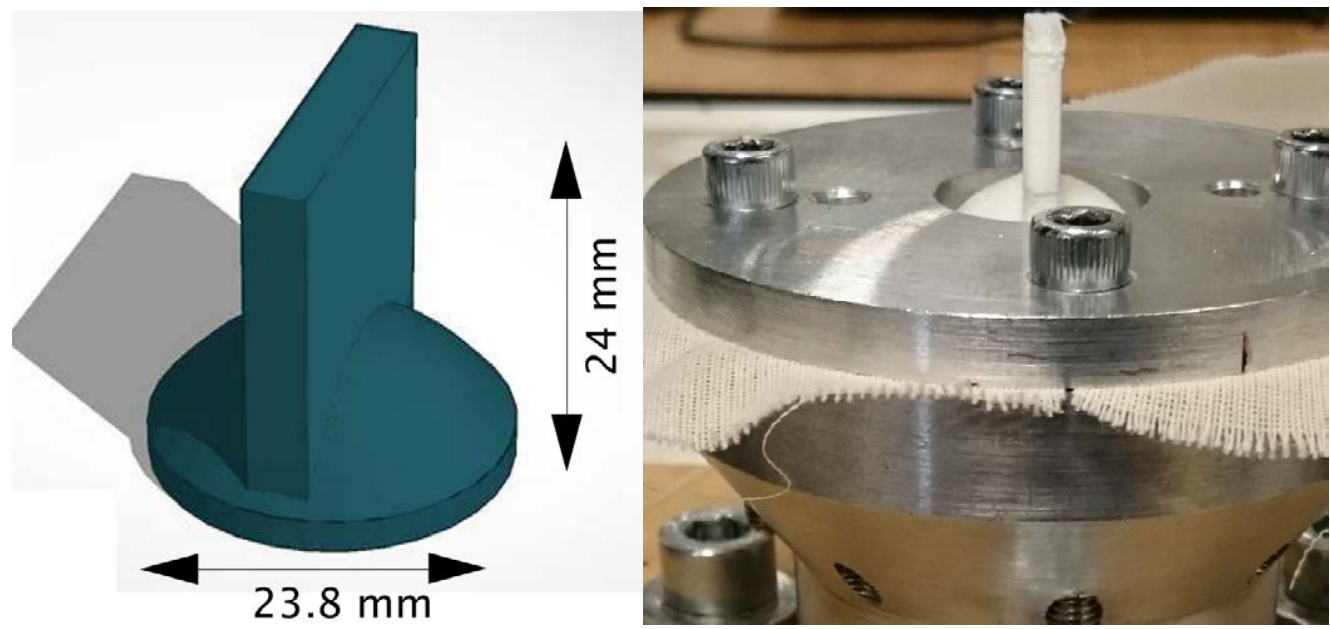

Figure 1: The dolly that is printed on the textile substrate. Left: Design of the dolly, Right: Dolly on the textile in a clamp.
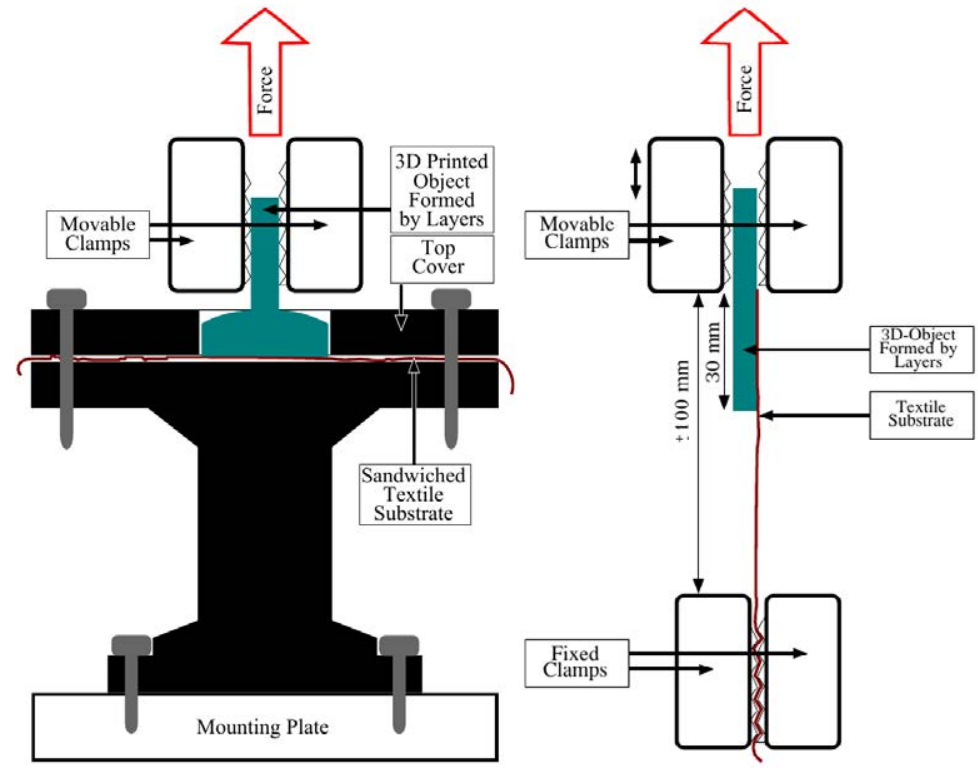

Figure 2: Left: The perpendicular tensile test. Right: Clamping of the specimen in the shear test.

\section{Materials}

The three proposed tests were used to characterize the adhesion of a $3 \mathrm{D}$ printed shape on six different textile substrates. As printing material, PLA was chosen for all the tests. For the first layers, the flow rate of the PLA material was reduced because the molten plastic penetrates into the textile fabric and hence cannot fill the entire volume under the nozzle. This reduction of flow rate was only done for the first layer, and was kept fixed for all materials used.

The testing methods were applied to determine if construction differences in fabrics have an influence on the adhesion properties. Preliminary tests showed that hairy textiles like wool prove to be unsuited as substrate for 3D FDM printing because the protruding fibres were flattened by the nozzle while printing, but return to their original shape afterwards. This effect deforms the still flexible printing material and results in a bad adhesion. PLA plastic was also found to provide no adhesion when printed on acrylated-coated cotton (neither did ABS plastic). As base material 100\% cotton was therefore selected.

Nine fabrics were woven with a cotton yarn (same for warp and weft) with different weave patterns and with different weft densities, as given in Table 1 . The fabrics came directly from the weaving loom that means they were not mercerised prior to printing.

\section{Results}

The three test methods were compared and it was shown how they can help in standardizing the research of 3D printing on textiles. Before testing the selected materials, tests were done on plain woven cotton fabric to determine the optimal initial height of the printer bed, the optimal fixation method of the textile to the printer bed (so that 


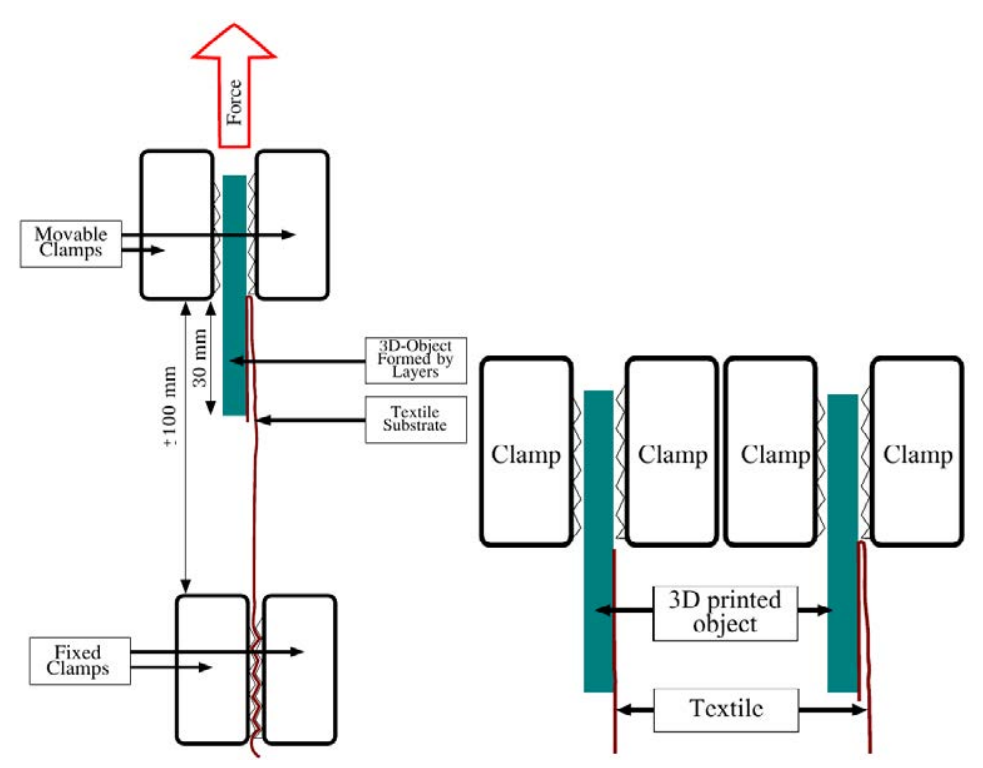

Figure 3: Left: The peel test. Right: Difference in attachment between the shear test and the peel test.
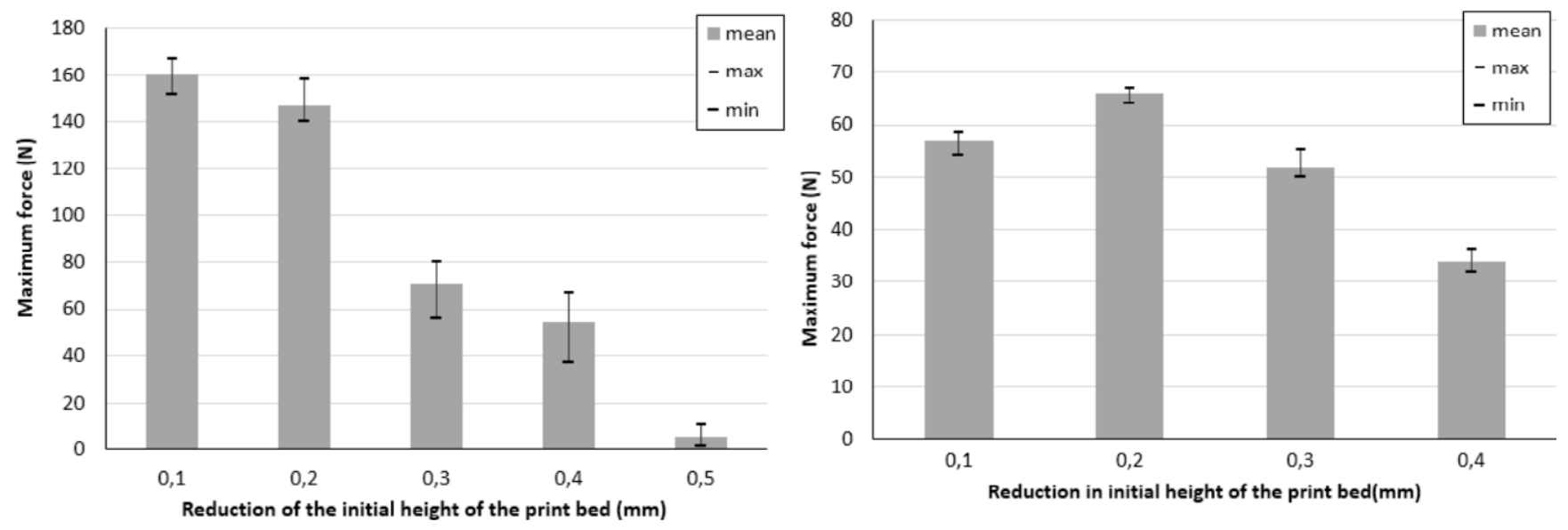

Figure 4: Left: Perpendicular tensile test on cotton fabric in terms of reduction of print bed height for an initial PLA layer of $0.3 \mathrm{~mm}$. Right: Peel test on cotton fabric in terms of reduction of print bed height for an initial PLA layer of $0.2 \mathrm{~mm}$. In both cases, the result shows a gap of $0.4 \mathrm{~mm}(=0.1+0.3$ and $0.2+0.2)$ between the nozzle and the printer bed gives the strongest adhesion. For the shear test, the fabric ruptured at this gap, so the adhesion bond is stronger than the fabric strength.

Table 1: Custom made fabrics for adhesion test.

\begin{tabular}{|l|l|l|}
\hline Fabric & Weave pattern & Weft density [yarns/cm] \\
\hline 4 & Plain & 18 \\
\hline 5 & Plain & 20 \\
\hline 6 & Twill 4 & 18 \\
\hline 7 & Twill 4 & 20 \\
\hline 8 & Satin 4 & 18 \\
\hline 9 & Satin 4 & 20 \\
\hline
\end{tabular}

it does not move during printing) and the influence of the printing temperature.

It was found that a gap of $0.4 \mathrm{~mm}$ between the print head and the printer bed was ideal for a cotton fabric of thickness 0.48 , as illustrated in Figure 4. A smaller distance caused scraping with the textile and removal of the textile from the printer bed, a higher distance caused a less good fusion of the plastic with the textile. As ways of fixing the textile to the printer bed, clamps, glue and double sided tape were tested. Tape was withheld because it gave the best results. As printing temperature, it was found that a too low temperature should be avoided. Best adhesion to the fabric started when a printing temperature of around $190^{\circ} \mathrm{C}$ was used.

With the optimal settings determined, the different samples were tested with the three test methods. The results were given in Figure 5. We found that for this material, with the printing settings as determined optimal, the shear and peel test mostly resulted in samples in which the fabric ruptured during the test before the printed object detached. We can conclude that this is the optimal behaviour for future use of 3D printing on textiles. However, the fabric "Plain 20" did come loose in the peel test, so is less suited as a substrate.

This is also evident in the perpendicular tensile test, which did 

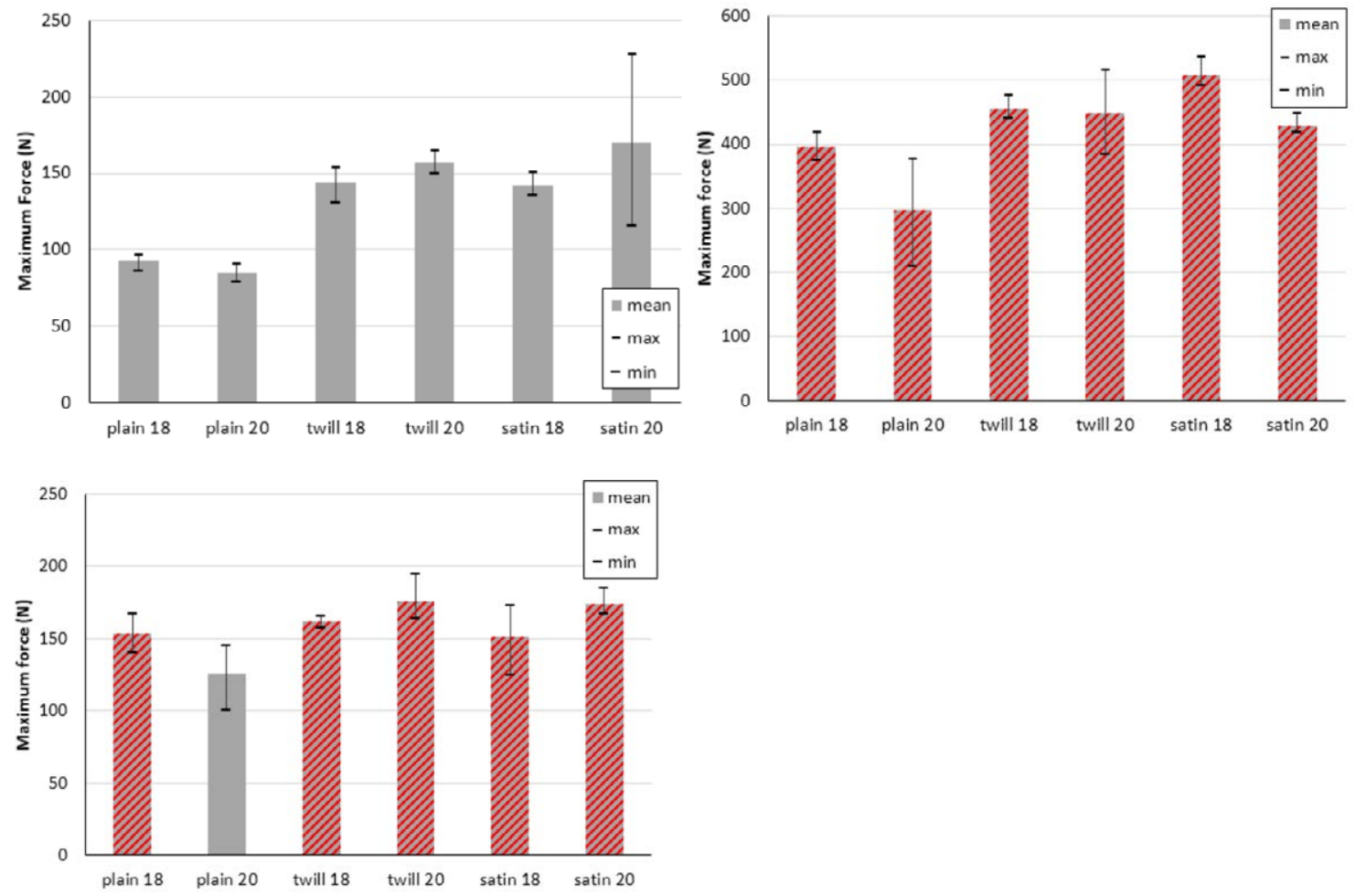

Figure 5: Top Left: Perpendicular tensile test, Top Right: Shear test, Bottom: Peel Test. Striped bars means that the fabric ruptured before the 3D printed object detached.

not show ruptured fabric in all six cases. The plain woven fabrics had least good adhesion, with the twill and satin giving dramatically better adhesion properties. For the plain fabric, increasing the weft density had no positive effect, whereas for the twill and satin fabrics, the force needed to detach the $3 \mathrm{~d}$ printed object slightly increased, meaning that the adhesion improved. It must be noted however that for the "Satin 20" fabric, the variance increased considerably. Overall, the "Twill 20" fabric was found to be the best textile substrate for printing 3D PLA structures, in terms of adhesion properties.

\section{Conclusions}

We can conclude that the three developed test methods were suitable to evaluate the adhesion properties of a $3 \mathrm{D}$ printed object on a textile substrate when printing a dedicated $3 \mathrm{D}$ object on the substrate first. This is a dolly for the perpendicular tensile test and a rectangular plate for the shear and peel test

Overall, we found the same trends in adhesion strength between the different tests, with the perpendicular tensile test the best test to determine overall adhesion strength, as it is less prone to rupturing the fabric before the test finishes. For applications that specifically will stress the shear or peel behaviour, we advise to perform a shear or peel test, after determining the optimal printing settings via the perpendicular tensile test. It was also proven that textile properties such as the weave pattern or the weft density will have an influence on the overall adhesion properties.

\section{References}

1. Gibson I, Rosen D, Stucker B (2014) Additive manufacturing technologies: 3D printing, rapid prototyping, and direct digital manufacturing, Springer, New York.
2. Sabantina L, Kinzel F, Ehrmann A, Finsterbusch K (2015) Combining 3D printed forms with textile structures-mechanical and geometrical properties of multi-material systems.

3. Pei E, Shen J, Watling J (2015) Direct 3D printing of polymers onto textiles: experimental studies and applications. Rapid Prototyp J 21: 556-571.

4. Rabe M (2015) 3D printing on textiles-new ways to textile surface modification.

5. Sanatgar R, Campagne C, Nierstrasz V (2017) Investigation of the adhesion properties of direct 3D printing of polymers and nanocomposites on textiles: Effect of FDM printing process parameters. Applied Surface Science 403: $551-563$.

\section{Author Affiliations}

${ }^{1}$ Centre for Textile Science and Engineering, Department MaTCh, Ghent University, Gent, Belgium

${ }^{2}$ Centre for Polymer and Material Technologies, Department MaTCh, Ghent University, Gent, Belgium

Submit your next manuscript and get advantages of SciTechnol submissions

* 80 Journals

* 21 Day rapid review process

* 3000 Editorial team

* 5 Million reader

* More than 5000

- Quality and quick review processing through Editorial Manager System

Submit your next manuscript at • www.scitechnol.com/submission 